\title{
A doença da Folha Verde do
Tabaco e suas implicações para a saúde do trabalhador
}

The green tobacco leaf disease and its implications for workers' health

FisiSenectus. Unochapecó Ano 6, n. 1 - Jan/Jun. 2018 p. 30-37

Daniele Cristina Stormovski. dani.storm@hotmail.com Fisioterapeuta, graduada pela Universidade do Contestado (UnC). Atua na área de Dermato-funcional.

Romário Henrique Basso. romario_henrique_b@hotmail.com Acadêmico de Enfermagem, na Universidade do Contestado (UnC). Atua no Grupo de Pesquisa em Saúde Coletiva e Meio Ambiente (NUPESC).

Renata Campos. renatacs@unc.br

Fisioterapeuta. Doutora em Ciências da Saúde pela Universidade de São Paulo (USP). Líder do Grupo de Pesquisa em Saúde Coletiva e Meio Ambiente (NUPESC). Docente dos cursos da área da Saúde na Universidade do Contestado.

\section{Resumo}

Introdução: A Doença da Folha Verde do Tabaco (DFVT) é a doença mais comum em fumicultores causada pela absorção da nicotina. A nicotina, presente na folha, é absorvida pela pele quando há contato com a folha molhada do tabaco, facilitada pela umidade da chuva ou pela umidade da transpiração. Assim que é absorvida, a nicotina entra na corrente sanguínea e é distribuída para todo o corpo, ocasionando sérios problemas à saúde do fumicultor. Objetivo: avaliar os efeitos da doença da Folha Verde do Tabaco na saúde do trabalhador. Material e métodos: foram avaliados 51 fumicultores ativos, por meio de um questionário semiestruturado relacionado a sintomatologia, ao uso de Equipamentos de Proteção Individual (EPIs) e as variáveis respiratórias, estas avaliaram a obstrução do fluxo e a capacidade inspiratória. Resultados: as variáveis respiratórias denotaram prejuízo da função pulmonar, evidenciado pelo Peak Flow, sendo de $291,96 \pm 117,42$ lpm ( $p=0,000)$, e da capacidade inspiratória, apresentando 2450,98 $\pm 1058,74 \mathrm{ml}(\mathrm{p}=0,01)$. Foi observado, também, que grande parte dos participantes nunca sentiram sintomatologia referente à Doença da Folha Verde e faziam uso do equipamento de proteção individual durante o cultivo. Conclusão: foram encontradas agravantes em relação à função respiratória dos fumicultores, indicando prejuízo para a saúde desses trabalhadores.

\section{Palavras-chave}

Agricultores; Intoxicação; Tabaco.

\section{Abstract}

Introduction: Green Tobacco Leaf Disease (DFVT) is the most common disease in this population and is caused by the absorption of nicotine. Nicotine is absorbed by the contact of the skin with the wet tobacco leaf, facilitated by rainfall moisture or moisture perspiration. Once absorbed, nicotine enters the bloodstream and

\section{Fisiłenectus}


is distributed throughout the body, causing serious problems to the worker's health. Aim: Evaluate the effects of the green tobacco leaf disease on the farmer's health. Material and methods: there were evaluated 51 tobacco farmers using a questionnaire about symptomatology, the use of individual protection equipment and respiratory variables, that estimates the outflow obstruction and the respiratory capacity. Results: the respiratory variables denote detriment on the pulmonary function and it has been made clear because the peak flow was $291,96+-117,42 \mathrm{Ipm}(\mathrm{p}=0,000)$ and the inspiratory capacity $2450,98+-1058,70 \mathrm{ml}(\mathrm{p}=0,01)$. The most of tobacco planters never felt simptomatology related to the green leaf disease and they are wearing the individual protection equipament during the cicle. Conclusion: it was found worsening on the levels of respiratory function of the tobacco planters, which indicates large detriment on their health.

\section{Keywords}

Farmers; Intoxication;Tobacco.

\section{Introdução}

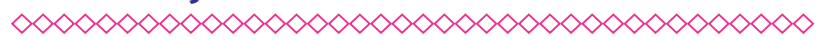

O Brasil é maior exportador mundial de tabaco desde 1993, e está em $2^{\circ}$ lugar no quesito de produção do fumo em folha. Sua maior produção se concentra no Sul do País, com destaque para o Rio Grande do Sul, com $50 \%$ da produção, seguido por Santa Catarina (27\%) e Paraná que vêm se destacando nos últimos sete anos, passando de $11 \%$ para $18 \%^{1}$.

A produção do tabaco é comum em pequenas propriedades rurais, desenvolvida principalmente pelo trabalho familiar, o qual é a preferência das empresas contratantes ${ }^{2}$. Como as propriedades que cultivam tabaco são familiares, marginalizadas em comparação com as condições urbanas, pouca orientação é dada ao fumicultor, deixando-os desassistidos em relação a sua saúde. Uma das doenças mais comuns em fumicultores é a Doença da Folha Verde do Tabaco (DFVT), que tem como causa a absorção da nicotina. Por ser solúvel em água, a nicotina é facilmente absorvida pelo contato da pele com a folha molhada do tabaco, pela umidade da chuva ou pela umidade da transpiração. Assim que é absorvida, a nicotina entra na corrente sanguínea e é distribuída para todo o corpo ${ }^{3}$. A DFVT tem como principais sintomas náuseas, vômitos, tontura, fraqueza e cefaleia ${ }^{4}$.

Essa doença é mais comum em produtores que trabalham com a folha molhada, na qual há um aumento na absorção da nicotina, do que com os que processam a folha seca ${ }^{4}$. A colheita e o transporte manual junto ao corpo favorecem o contato com a seiva. Além disso, a possibilidade de ocorrer cortes na pele potencializa a doença ${ }^{3}$.

Oliveira et al. ${ }^{4}$ relatam que a intoxicação pela DFVT pode ser minimizada se o trabalhador usar equipamentos de segurança individual, como luvas e roupas impermeáveis. Caso o trabalhador não disponha de luvas, deve fazer a higienização constante das mãos, a qual diminui a presença de nicotina nas mãos, e consequentemente a absorção.

Uma característica importante da DFVT é que essa doença pode ser autolimitante, possibilitando que os produtores se recuperem em dois ou três dias. Porém, os sintomas podem se agravar, causando desidratação e necessidade de atendimento médico ${ }^{5}$.

A nicotina apresenta consequências em todos os sistemas do corpo. No sistema cardiovascular, causa aumento da pressão arterial, aumento da frequência cardíaca, e vasoconstrição. No sistema gastrointestinal, estimula o aumento do tônus e da atividade motora do intestino. No sistema endócrino, contribui para a restrição hídrica e liberação do hormônio antidiurético, causando diminuição do fluxo urinário ${ }^{6}$. Nas terminações nervosas, ela impulsiona a liberação dos neurotransmissores como acetilcolina, serotonina, glutamato, dopamina e ácido gama-aminobutírico ${ }^{7}$. No sistema reprodutor masculino, a nicotina causa diminuição na função normal do espermatozoide, reduzindo a capacidade de fertilização. Em mulheres, também ocorrem alterações reprodutivas e aceleração na menopausa ${ }^{8}$.

Durante a gravidez, o contato com a nicotina pode causar antecipação ao parto, taquicardia 
fetal, baixo peso ao nascer, problemas respiratórios e alterações no desenvolvimento neurológico da criança?.

Esta pesquisa estudou os efeitos da doença da folha verde do tabaco na saúde do trabalhador.

\section{Materiais e métodos}

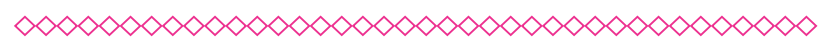

Trata-se de uma pesquisa descritiva, transversal, desenvolvida com fumicultores nos municípios de Campo do Tenente/PR e Itaiópolis/SC. Este projeto seguiu todos os preceitos éticos e legais da resolução 466/12 do Conselho Nacional de Saúde, sendo aprovado pelo Comitê de Ética e Pesquisa da Universidade do Contestado, sob parecer $\mathrm{n}^{0} 1.343 .859$.

Os critérios de inclusão para a pesquisa foram indivíduos que trabalhassem com o cultivo da Folha Verde do Tabaco, desde o plantio até a colheita, na faixa etária de 21 à 59 anos e que concordassem em participar da pesquisa assinando o Termo de Consentimento Livre e Esclarecido (TCLE). Os critérios de exclusão foram tabagistas e fumicultores com histórico prévio de doença pulmonar crônica e câncer pulmonar.

No encontro entre fumicultor e o pesquisador, que ocorreu na residência do próprio fumicultor, foi esclarecida a finalidade da pesquisa e após autorização, foi aplicado o questionário semiestruturado, com questões relacionadas a identificação do voluntário (idade, sexo e altura), a presença de sintomas durante o cultivo, o uso de equipamentos de segurança, o tempo de trabalho no cultivo e a escolaridade. Toda a fase de coleta de dados foi realizada por um único pesquisador para evitar o viés nesta etapa da pesquisa.

Após a etapa inicial, deu-se continuidade à coleta de dados pelo segundo questionário - o Mini Exame do Estado Mental (MEEM), o qual é composto por questões com o objetivo de avaliar funções cognitivas específicas como a capacidade de memória, linguagem, atenção, construção visual, recordatória, cálculo, orientação espacial e corporal. o escore máximo atribuído a cada tarefa completada com sucesso é 30.0 ponto de corte é frequentemente ajustado para o nivel educacional porque um único corte pode perder casos entre pessoas com escolaridade mais elevada e gerar falsos positivos entre aqueles com menor escolaridade. 0 ponto de corte 24 mostrou-se excelente para pessoas com escolaridade acima de nove anos, enquanto o corte 17 foi ótimo para aqueles com menor escolaridade ${ }^{10}$. Em contrapartida, o estudo de UhImann e Larson ${ }^{11}$ descreveu ponto de corte 20/21 para aqueles com 8 a 9 anos de escolaridade, 22/23 para aqueles com 10 a 12 anos e 23/24 para aqueles com mais anos de educação.

Além da avaliação das funções cognitivas, foram analisadas as variáveis respiratórias, compostas por:

a) Obstrução ao fluxo aéreo expiratório, que foi medido pelo Peak Flow Meter. Os fumicultores foram instruídos a realizar uma inspiração máxima seguida de uma expiração rápida e vigorosa, não podendo tossir ou prolongar por mais de dois segundos ${ }^{12}$. 0 teste foi realizado três vezes e o maior valor foi registrado;

b) A capacidade inspiratória, que foi avaliada pelo Inspirômetro de Incentivo da marca Voldyne ${ }^{\circledR 13}$. O valor de referência descrito na literatura é de $3.000 \mathrm{ml}^{14}$. Os fumicultores foram instruídos a realizarem uma expiração até a capacidade residual funcional e após uma inspiração lenta e mais profunda possível ${ }^{15}$.

Ao final do uso de cada equipamento para a avaliação respiratória, houve o processo de desinfeção química por álcool 70\% com friç̧ão e uso de bucais individuais, para assegurar a higiene dos equipamentos ao próximo entrevistado.

Para a análise estatística, foi utilizado o programa SPSS 21. Para as variáveis numéricas, empregou-se a média e o desvio padrão. Para as variáveis categóricas, foi utilizada a frequência. 0 teste de Kolmogorov - Smirnov foi realizado para avaliar a distribuição normal da amostra. Dados comparativos foram executados pelo teste $\mathrm{t}$ de Student, adotando nível de significância se $p<0,05$.

\section{Resultados}

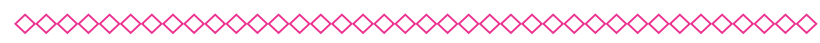

A pesquisa foi realizada com 51 fumicultores, sendo $54,9 \%$ do sexo masculino e $45,1 \%$ do sexo 
feminino, na faixa etária de 21 a 59 anos, sendo $55 \%$ com grau de escolaridade ensino fundamental incompleto e $41,2 \%$ trabalham no cultivo a mais de 20 anos (Tabela $\mathbf{1}$ ).

Na Tabela 2, observa-se que, durante o cultivo, a sintomatologia da DFVT foi caracterizada algumas vezes pela sintomatologia relacionada ao contato com a nicotina potencializada pela folha de tabaco úmida, apresentando tontura (33,3\%), vômito $(33,3 \%)$, dor de cabeça $(23,5)$ e fraqueza $(11,8 \%)$.

Grande parte dos fumicultores faz o uso de equipamentos de proteção individual (EPI) durante o cultivo do tabaco, tais como macacão, luvas, botas (Tabela 3 ).

0 valor obtido por meio do Peak Flow Meter (Gráfico 1) foi de 291,96 $\pm 117,42$ Ipm e o valor predito, calculado pela idade e altura, seria de $528,67 \pm 102,71(p=0,000)$.

0 valor obtido da CI foi de $2.450,98 \pm 1.058,74$ $\mathrm{ml}$, sendo queo valor de referência comparativo descrito na literatura é de $3.000 \mathrm{ml}^{14}$, tornando essa comparação estatisticamente significativa $(p=0,01)$ (Gráfico 2).

Na avaliação do sistema nervoso, não foram encontradas alterações cognitivas nos fumicultores avaliados. Para os agricultores com ensino fundamental incompleto, o MEEM teve uma pontuação de 27,28. Para os fumicultores com fundamental completo, o escore foi de 27,50. Para a escolaridade ensino médio incompleto teve uma pontuação de 29,50 e para o médio completo 29,08. Dessa forma, pela pontuação, não houve alteração.

\section{Discussão}

$\infty \times \infty \times \infty \times \infty \times \infty \times \infty \times \infty \times \infty \times \infty \times \infty \times \infty \times \infty \infty$

A grande maioria dos jovens com idade de aproximadamente 25 e 30 anos tem manifestado o desejo em continuar sua vida profissional na fumicultura. Isso é fortalecido pelo Programa Nacional de Fortalecimento da Agricultura Familiar que tem oferecido crédito para jovens agricultores. Por meio do avanço e da aplicabilidade da tecnologia e ciência no campo, espera-se que os jovens permaneçam nas atividades de seus pais, perpetuando esse ciclo familiar. No contraponto, Arenhardt ${ }^{16}$ relata que os jovens estão se preparando para deixar o meio rural, na busca do seu futuro profissional.

Há evidências de que os trabalhadores do sexo masculino com idade entre 15 e 19 anos tiveram maior mortalidade por intoxicação por agrotóxi$\cos ^{17}$. 0 contato com a nicotina pode causar a intoxicação e trazer sintomatologia característica. Os sintomas estão associados ao estímulo ou à inibição de receptores no sistema nervoso central, causando um quadro clínico de vômitos, náuseas, tonturas e cefaleia ${ }^{18}$. Esse quadro muitas vezes é subnotificado, por ser facilmente confundido com sintomas de intoxicação alimentar. No presente estudo, observou-se que a grande maioria dos trabalhadores já tiveram algum sinal de intoxicação.

Os EPIS são fundamentais para a proteção da saúde do trabalhador e evitar as intoxicações ocupacionais. Dessa forma, as roupas impermeáveis são indicadas para evitar o contato com o agrotóxico e com a nicotina da folha do tabaco. Contudo, na maioria das vezes deixam de ser usadas, devido ao calor excessivo que elas causam. É importante ressaltar que, quando são lavadas, essas roupas perdem a ação impermeável, devendo ser descartadas após o uso. Esse fato eleva o custo, por isso, os fumicultores costumam reutilizá-las como forma de minimizar os gastos, surgindo mais um agravo para a saúde do trabalhador.

É interessante notar que, no presente estudo, houve o relato por parte dos fumicultores que 0 uso dos EPIs é frequente. Estudo de Arcury et al. ${ }^{19}$ enfatiza que as roupas de proteção minimizam a absorção da nicotina pela pele e, consequentemente, as intoxicações; porém, muitas vezes, os EPIs não são utilizados por prejudicarem o processo da colheita na percepção dos fumicultores.

O contato com a nicotina da Folha Verde do Tabaco também pode causar alterações relevantes na função respiratória, como a diminuição da capacidade residual funcional, no volume corrente e na complacência pulmonar, hipoventilação alveolar, retenção de dióxido de carbono, aumento da resistência ao fluxo aéreo da frequência respiratória e na mecânica respiratória, com alteração na movimentação do diafragma e da caixa torácica, aumentando o trabalho muscular respiratório. o pico de fluxo expiratório é importante para diagnóstico, monitorização e controle da função 
respiratória. Sua variação pode ser utilizada para documentar a obstrução do fluxo aéreo. No presente estudo, houve diminuição acentuado de seus valores, indicando importante limitação expiratória. Este teste é importante para indicar a deterioração da função pulmonar no decorrer do tempo ${ }^{20}$, assim como a capacidade inspiratória máxima, que é definida como volume máximo de ar inspirado. Sua redução é uma anormalidade bastante evidente em paciente com fraqueza muscular respiratória ou com alterações de mecânica pulmonar que levam à sobrecarga dos músculos respiratórios. A diminuição do volume inspirado pode causar hipoventilação, prejuízo da respiração profunda, padrão respiratório monótono e diminuição da eficácia da tosse ${ }^{21}$. Observou-se uma redução significativa da capacidade inspiratória nos fumicultores estudados, indicando alteração no processo ventilatório da amostra.

\section{Conclusão}

$\infty \times \infty \times \infty \times \infty \times \infty \times \infty \times \infty \times \infty \times \infty \times \infty \times \infty \times \infty \times \infty)$

Embora a literatura indique que a sintomatologia causada pela Doença da Folha Verde do Tabaco seja comum na prática da fumicultura, neste estudo verificamos que, entre os fumicultores avaliados, grande parte nunca apresentou a sintomatologia, o que pode estar relacionado ao uso constante dos EPIs. Também foram encontrados agravantes em relação à função respiratória dos fumicultores, com prejuízos à saúde, indicativo de uma intoxicação crônica pela prática da fumicultura. Entretanto, estudos nesta linha devem ser conduzidos na tentativa de comprovar o nexo causal.

\section{Referências}

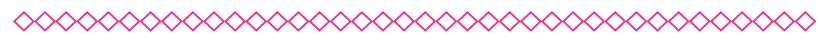

1. Sinditabaco [homepage na Internet]. Dimensão do setor [acesso em 09 ago 2018]. Disponível em: http://sinditabaco.com.br/sobre-osetor/ dimensoes-do-setor/

2. Almeida GEG. Fumo: servidão moderna e violações de direitos humanos. Curitiba: Terra de Direitos; 2005.
3. McBride JS, Altman DG, Klein M, White W. Green tobacco sickness. North Carolina: Tobacco Control. 1998; 7:294-298.

4. Oliveira PPV, Sihler CB, Moura L, Malta DC, Torres MCA, Lima SMCP, et al. First reported outbreak of green tobacco sickness in Brazil. Cad. Saúde Pública. 2010; 26(12): 2263-2269.

5. Arcury TA, Quandt SA, Preisser JS. Predictors of incidence and prevalence of green tobacco sickness among Latino farmworkers in North Carolina, USA. J Epidemiol Community Health. 2001; 55:818-824.

6. Furtado RD. Implicações anestésicas do tabagismo. Rev Bras Anestesiol. 2002; 52:354367.

7. Rose JE, Behm FM, Westman EC, Mathew RJ, London ED, Turkington TG, et al. PET Studies of the Influences of Nicotine on Neural Systems in Cigarette Smokers. Am J Psychiatry. 2003; 160(2):323-333.

8. Reinberg S. HealthDay [homepage na Internet]. Smoking reduces a man's fertility. Disponível em: https://consumer.healthday.com/infertilityinformation-22/infertility-news-412/smokingreduces-a-man-s-fertility-528575.html

9. Muler JS, Antunes M, Behle I, Teixeira L, Zielinsky P. Efeitos agudos do fumo sobre a hemodinâmica da circulação fetomaternoplacentária. Arq Bras Cardiol. 2002; 78(2):148-151.

10. Bertolucci PH, Okamoto IH, Brucki SM, Siviero MO, Toniolo Neto J, Ramos LR. Applicability of the CERAD neuropsychological battery to Brazilian elderly. Arq Neuro-psiquiatr. 2001; 59(3A):532536.

11. UhImann RF, Larson EB. Effect of education on the mini-mental state examination as a screening test for dementia. J Am Geriatr Soc. 1991;39(9):876-880.

12. Freitas FS, Ibiapina CC, Alvim CG, Britto RR, Parreira VF. Relação entre força de tosse e nível funcional em um grupo de idosos. Rev Bras Fisioter. 2010; 14(6):470-476. 
13. Ferreira FS, Weber $P$, Corrêa ECR, Milanesi JM, Borin GS, Dias MF. Efeito da fisioterapia sobre os parâmetros ventilatórios e a dinâmica tóracoabdominal de crianças respiradoras bucais. Fisioter Pesqui. 2012; 19(1):8-13.

14. Guyton AC, Hall JE. Fisiologia Humana. 6. ed. São Paulo: Guanabara,1998.

15. Schettino RC, Jotta LMGN, Cassali GD. Função pulmonar em mulheres com câncer de mama submetidas à radioterapia: um estudo piloto. Fisioter Pesqui [periódicos da Internet]. 2010;17(3):248-252.

16. Arenhardt DL, Souza MA, Franchi TS. O jovem rural e seu olhar sobre o futuro: a busca por melhor condição de vida nos centros urbanos. Contribuciones A Las Ciencias Sociales [periódicos na Internet]. 2014. Disponível em: http://www. eumed.net/rev/cccss/28/juventude-rural.html

17. Wesseling C, Castillo L, Elinder CG. Pesticide poisonings in Costa Rica. Scand J Work Environ Health. 1993;19(4):227-35.
18. Arcury TA, Quandt SA, Garcia DI, Preisser JS, Norton D, Rao P. A clinic-based, case-control comparison of Green tobacco sickness among minority farmworkers: clues for prevention. South Med J. 2002; 95(9):1008-1011.

19. Arcury $T A$, Vallejos $Q M$, Schulz MR, Feldman SR, Fleischer AB, Verma A, et al. Green tobacco sickness and skin integrity among migrant latino farmworkers. Am J Ind Med. 2008;51(3):195-203.

20. IV Diretrizes Brasileiras para o Manejo da Asma. J Bras Pneumol. 2006; 32(7):S447-S474.

21. Dias CM, Plácido TR, Ferreira MFB, Guimarães FS, Menezes SLS. Inspirometria de incentivo e breath stacking: repercussões sobre a capacidade inspiratória em indivíduos submetidos à cirurgia abdominal. Rev Bras Fisioter. 2008; 12(2):94-99. 


\section{Anexos}

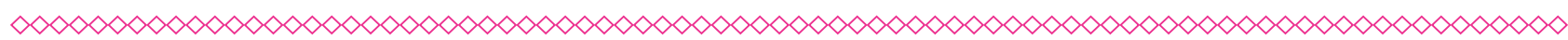

Tabela 1 - Dados gerais dos fumicultores

\begin{tabular}{lcc} 
Variável & $\mathrm{n}$ & $\%$ \\
Sexo & & \\
Masculino & 28 & 54,9 \\
Feminino & 23 & 45,1 \\
Escolaridade & & \\
Fundamental incompleto & 28 & 55 \\
Fundamental completo & 2 & 3,9 \\
Médio incompleto & 9 & 17,6 \\
Médio completo & 12 & 23,5 \\
Tempo de cultivo & & \\
Menos de 1 ano & 6 & 11,8 \\
Entre 5 e 10 anos & 19 & 37,3 \\
Entre 15 e 20 anos & 5 & 9,7 \\
Mais de 20 anos & 21 & 41,2 \\
\hline
\end{tabular}

(clique para voltar ao texto)

Tabela 2 - Sintomatologia causada pela DFVT

\begin{tabular}{cccccc} 
Sintomas & Nunca & Raramente & Às vezes & Frequentemente & Sempre \\
Tontura (\%) & 47,1 & 11,8 & 33,3 & 0 & 7,8 \\
Vômito (\%) & 41,2 & 21,6 & 33,3 & 2 & 2 \\
Cefaleia (\%) & 49 & 13,7 & 23,5 & 0 & 13,7 \\
Fraqueza (\%) & 70,6 & 11,8 & 11,8 & 2 & 3,9 \\
\hline
\end{tabular}

DFVT= Doença da Folha Verde do Tabaco

(clique para voltar ao texto)

Tabela 3 - Uso de EPI

\begin{tabular}{cccccc} 
Equipamento & Nunca & Raramente & Às vezes & Frequentemente & Sempre \\
\hline Macacão (\%) & 29,4 & 0 & 11,8 & 2 & 56,9 \\
Luvas (\%) & 19,6 & 0 & 11,8 & 0 & 68,6 \\
Botas (\%) & 19,6 & 0 & 2 & 0 & 78,4 \\
Máscara (\%) & 64,7 & 2 & 5,9 & 0 & 27,5 \\
Chapéu (\%) & 2 & 0 & 3,9 & 2 & 92,2 \\
\hline
\end{tabular}


Gráfico 1 - Dados apresentados para o Peak flow obtido pelo paciente e o predito de acordo com a altura e idade dos entrevistados

$$
\text { Peak Flow }
$$

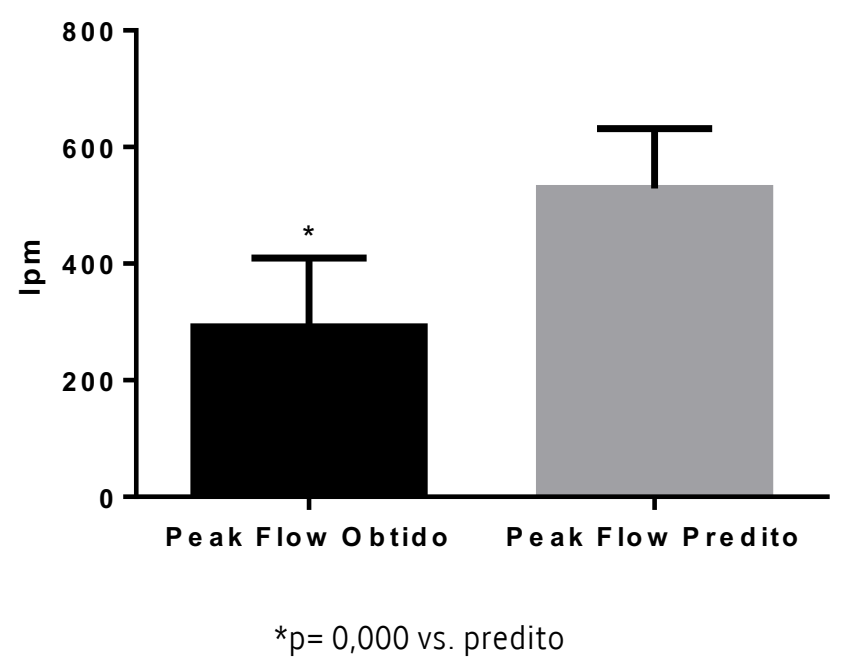

(clique para voltar ao texto)

Gráfico 2 - Dados de Capacidade inspiratória (CI) obtida pelos fumicultores comparado com o valor mínimo estabelecido pela literatura ${ }^{13}$

Capacidade Inspiratória

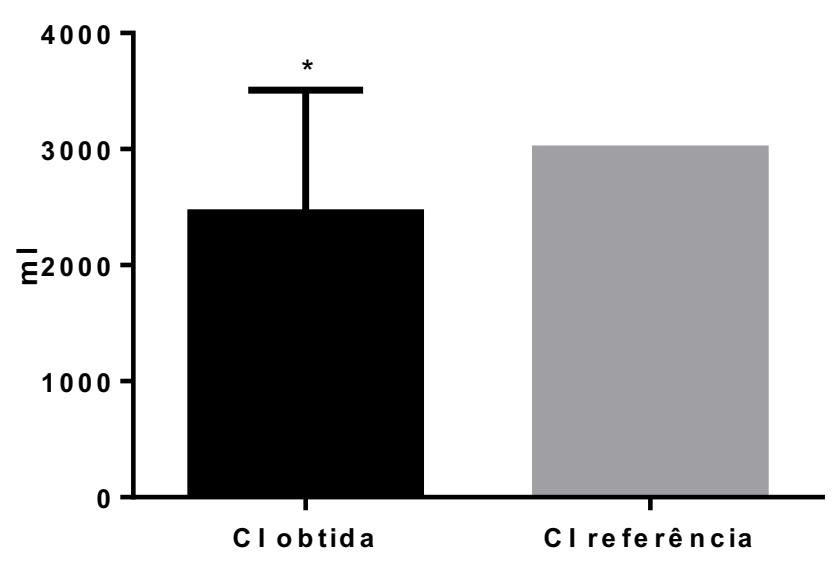

${ }^{*} p=0,01$ vs. referência 